\title{
Erratum: Parametrized-4.5PN TaylorF2 approximants and tail effects to quartic nonlinear order from the effective one body formalism [Phys. Rev. D 95, 124001 (2017)]
}

Francesco Messina and Alessandro Nagar

(Received 1 May 2018; published 29 May 2018)

DOI: 10.1103/PhysRevD.97.109902

A further error was spotted in the parametrized 5PN term of the total flux. To obtain it consistently by expanding the EOB flux one should also include the even-parity $l=7$ multipoles - that is, $(7,7),(7,5),(7,3)$, and $(7,1)$ - that were omitted by mistake. Note, however, that the 5.5PN term only depends on the $\ell=6$ modes, so that term (modulo a typo) was computed correctly. Once the $\ell=7$ modes are included, Eq. (21) of our paper is corrected as

$$
\begin{aligned}
\hat{\mathcal{F}}_{5 \mathrm{PN}}= & \left\{-\frac{1}{1-3 \nu}\left[\frac{2500861660823683}{2831932303200}-\frac{10608155067013217101261}{1904961566258150400} \nu+\frac{24823886833955583083459}{3265648399299686400} \nu^{2}\right.\right. \\
& +\frac{83916740839405609479263}{68578616385293414400} \nu^{3}+\frac{31282558612344304720193}{3809923132516300800} \nu^{4}-\frac{163716515443482656797}{35168521223227392} \nu^{5} \\
& +\frac{1247153383060759670851}{1714465409632335360} \nu^{6}+\gamma_{\mathrm{E}}\left(-\frac{916628467}{7858620}+\frac{3232420865}{3667356} \nu-\frac{12533953163}{9168390} \nu^{2}-\frac{2084604421}{3056130} \nu^{3}\right) \\
& +\pi^{2}\left(\frac{424223}{6804}-\frac{520672457}{1354752} \nu+\frac{1244952467}{4064256} \nu^{2}+\frac{888697001}{1016064} \nu^{3}-\frac{36678805}{677376} \nu^{4}\right) \\
& +\left(\frac{83217611}{1122660}-\frac{11385383927}{18336780} \nu+\frac{12117535706}{4584195} \nu^{2}-\frac{1327348993}{305613} \nu^{3}\right) \log (2) \\
& +\left(-\frac{47385}{196}+\frac{1405755}{784} \nu-\frac{2827305}{784} \nu^{2}+\frac{236925}{196} \nu^{3}\right) \log (3) \\
& \left.+\left(-\frac{916628467}{15717240}+\frac{3232420865}{7334712} \nu-\frac{12533953163}{18336780} \nu^{2}-\frac{2084604421}{6112260} \nu^{3}\right) \log (x)\right] \\
& +\left(-\frac{114}{7}+\frac{55}{7} \nu\right) \nu c_{22}^{4 \mathrm{PN}}(\nu)+4 \nu c_{22}^{5 \mathrm{PN}}(\nu)+\frac{1}{9} \nu(1-4 \nu) c_{21}^{4 \mathrm{PN}}+\left(-\frac{1}{56}+\frac{151}{756} \nu-\frac{97}{189} \nu^{2}\right) \nu c_{21}^{3 \mathrm{PN}}(\nu) \\
& +\nu(1-4 \nu)\left(\frac{1}{1344} c_{31}^{4 \mathrm{PN}}(\nu)+\frac{3645}{448} c_{33}^{4 \mathrm{PN}}(\nu)\right)+\nu(1-3 \nu)^{2}\left(\frac{10}{21} c_{32}^{3 \mathrm{PN}}(\nu)+\frac{10240}{567} c_{44}^{3 \mathrm{PN}}(\nu)+\frac{40}{3969} c_{42}^{3 \mathrm{PN}}(\nu)\right) \\
& \left.-\nu(1-2 \nu)^{2}(4 \nu-1)\left(\frac{729}{560} c_{43}^{2 \mathrm{PN}}+\frac{1}{35280} c_{41}^{2 \mathrm{PN}}(\nu)+\frac{48828125}{1216512} c_{55}^{2 \mathrm{PN}}(\nu)+\frac{2187}{45056} c_{53}^{2 \mathrm{PN}}(\nu)+\frac{1}{127733376} c_{51}^{2 \mathrm{PN}}(\nu)\right)\right\} x^{5} .
\end{aligned}
$$

Similarly, Eq. (22) of our paper should also be amended as

$$
\begin{aligned}
\hat{\mathcal{F}}_{5.5 \mathrm{PN}}= & \pi\left\{\frac{2 \nu}{9}(1-4 \nu) c_{21}^{3 \mathrm{PN}}(\nu)+\frac{6058253667029 \nu^{4}}{64076044032}+\frac{70029960211823 \nu^{3}}{85434725376}+\frac{100004171503889 \nu^{2}}{160190110080}\right. \\
& +\nu\left(16 c_{22}^{4 \mathrm{PN}}(\nu)-\frac{138140205552539713}{25630417612800}\right)+\frac{8399309750401}{101708006400}+\pi^{2}\left(\frac{1015201 \nu}{193536}-\frac{1605601 \nu^{2}}{48384}\right) \\
& +\gamma_{\mathrm{E}}\left(\frac{2781341 \nu}{4410}+\frac{177293}{1176}\right)+\left(\frac{2360189 \nu}{4410}+\frac{8521283}{17640}\right) \log (2)+\left(\frac{142155 \nu}{196}-\frac{142155}{784}\right) \log (3) \\
& \left.+\left(\frac{2781341 \nu}{8820}+\frac{177293}{2352}\right) \log (x)\right\} x^{11 / 2},
\end{aligned}
$$


with $\mathrm{a}+$ sign that replaces the $\times$ that was incorrectly introduced in front of the fifth term in the published version and that was incorrectly replaced by a - sign in the first erratum. These results are now fully consistent with the 5.5PN test-particle fluxes of Tanaka et al. [1] [Eq. (3.1) therein]. In the correct formulas above, some " $4 \mathrm{PN}$ " labels on the $c_{21}$ coefficient have been substituted by the correct relative 3PN order ones. In the paper, the residual relativistic amplitude of the $(2,1)$ multipole (Eq. 8) is also wrong (though it was correct in the MATHEMATICA notebook used). The correct form of this object, in which the relative $3 \mathrm{PN}$ coefficient also appears, is given by

$$
\begin{aligned}
\rho_{21}(x ; \nu)= & \rho_{21}^{2 \mathrm{PN}}(x ; \nu)+\left(\frac{7613184941}{2607897600}-\frac{107}{105} \text { eulerlog }_{1}(x)+\nu c_{21}^{3 \mathrm{PN}}(\nu)\right) x^{3} \\
& +\left(-\frac{1168617463883}{911303737344}+\frac{6313}{5880} \text { eulerlog }_{1}(x)+\nu c_{21}^{4 \mathrm{PN}}(\nu)\right) x^{4} .
\end{aligned}
$$

Finally, the correct TaylorF2 phase coefficient at 4PN is

$$
\begin{aligned}
\varphi_{8}= & {[1-\log (\pi f M)]\left[c_{21}^{3 \mathrm{PN}}\left(\frac{40 \nu}{81}-\frac{160 \nu^{2}}{81}\right)+\frac{160 c_{22}^{4 \mathrm{PN}} \nu}{9}-\frac{369469478275 \nu^{4}}{16019011008}+\frac{510041481025 \nu^{3}}{13106463552}\right.} \\
& +\left(\frac{300600673165997}{576684396288}-\frac{399545 \pi^{2}}{27216}\right) \nu^{2}+\left(-\frac{5679872289503527}{1281520880640}-\frac{5322928 \gamma}{3969}+\frac{9302215 \pi^{2}}{54432}\right. \\
& \left.-\frac{1420688 \log (2)}{441}+\frac{26325 \log (3)}{49}\right) \nu-\frac{90490 \pi^{2}}{567}-\frac{36812 \gamma}{189}+\frac{2550713843998885153}{830425530654720}-\frac{26325 \log (3)}{196} \\
& \left.-\frac{1011020 \log (2)}{3969}\right]+\left(\frac{2661464 \nu}{11907}+\frac{18406}{567}\right) \log ^{2}(\pi f M) .
\end{aligned}
$$

The conclusions of the paper are not affected by these corrections.

[1] T. Tanaka, H. Tagoshi, and M. Sasaki, Prog. Theor. Phys. 96, 1087 (1996). 